\title{
Detrended Fluctuation Analysis and Hough Transform Based Self-Adaptation Double-Scale Feature Extraction of Gear Vibration Signals
}

\author{
JiaQing Wang, Han Xiao, Yong Lv, Tao Wang, and Zengbing Xu \\ Hubei Province Key Lab of Machine Transmission and Manufacturing Engineering, Wuhan University of Science and Technology, \\ P.O. Box 222, Wuhan, Hubei 430081, China
}

Correspondence should be addressed to Han Xiao; coolxiaohan@163.com

Received 27 July 2015; Revised 2 December 2015; Accepted 7 December 2015

Academic Editor: Evgeny Petrov

Copyright (c) 2016 JiaQing Wang et al. This is an open access article distributed under the Creative Commons Attribution License, which permits unrestricted use, distribution, and reproduction in any medium, provided the original work is properly cited.

\begin{abstract}
This paper presents the analysis of the vibration time series of a gear system acquired by piezoelectric acceleration transducer using the detrended fluctuation analysis (DFA). The experimental results show that gear vibration signals behave as double-scale characteristics, which means that the signals exhibit the self-similarity characteristics in two different time scales. For further understanding, the simulation analysis is performed to investigate the reasons for double-scale of gear's fault vibration signal. According to the analysis results, a DFA double logarithmic plot based feature vector combined with scale exponent and intercept of the small time scale is utilized to achieve a better performance of fault identification. Furthermore, to detect the crossover point of two time scales automatically, a new approach based on the Hough transform is proposed and validated by a group of experimental tests. The results indicate that, comparing with the traditional DFA, the faulty gear conditions can be identified better by analyzing the double-scale characteristics of DFA. In addition, the influence of trend order of DFA on recognition rate of fault gears is discussed.
\end{abstract}

\section{Introduction}

Generally, the gear transmission systems are characterized with periodic behaviors. However, the defects of gears, bearings, or transmission shafts may cause the nonlinear vibration. The gearbox vibration signals captured by the sensors are complicated, nonlinear, and nonstationary $[1,2]$. Many researchers verified that the vibration time series of the gear transmission systems exhibit nonlinearity and selfsimilarity $[1,3,4]$. Therefore, a lot of the nonlinear time series analysis methods and several nonlinear characteristic quantities such as fractal dimension [5], entropy [6], and the Lyapunov exponent [7] have been employed to detect the faults. Though these nonlinear based methods may be suitable to analyze the nonlinear characteristics of vibration signals, they are difficult to obtain the more accurate results without considering the real scale related features of the time series which are characterized with multiexponents or nonlinear parameters.
In the recent years, the fractal or multifractal time series have been observed in many fields, such as geophysics time series, medical time series, and technical time series [8]. The traditional approaches for the fractal analysis, such as Hurst's rescaled-range analysis $(R / S)$ [9] and fluctuation analysis (FA) [8], always assume the time series as the stationary data without considering the possible fluctuation caused by some reasons. The methods for the nonstationary time series include the wavelet analysis, the discrete wavelet transform (WT), and the detrended fluctuation analysis (DFA) [8]. The DFA which was first introduced by Peng et al. in 1992 is a new Hurst exponent calculation method [10] based on the random walk theory. Basically, it represents a detrending version of fluctuation analysis (FA), which is more reliable and suitable for analyzing the nonstationary signal compared to the $R / S$ or the FA analysis. This is because the DFA can remove the external polynomial trends of the differential orders in order to obtain the accurate intrinsic statistical characteristics from the time series. One advantage of the DFA is that 
it can detect the long-range correlations embedded in the seemingly nonstationary time series and also avoid the spurious detection of the apparent long-range correlations which are an artifact of nonstationarity. The DFA has been widely applied to various fields, such as meteorology [11], materials science [12], finance [13], biological signals [14], and hydrograph [15]. Several modified DFA methods have also been proposed [8].

The DFA was also used in equipment fault diagnosis. de Moura et al. [16] employed the DFA and the principal component analysis (PCA) to the cluster analysis of gear faults. Instead of using the long-range correlation or scale exponents of time series, the idea of Moura's method is to use the fluctuation function as a mapping function from data space to characteristic space. Afterwards, De Moura et al. [17] used the DFA to analyze the bearing fault. Sridhar et al. [18] combined the EEMD with DFA to denoise the noise-corrupted signal. The DFA are used to determine the noise components in IMFs. Through the DFA, the crossover phenomenons are found in finance [19], meteorology [20], medical science [21], and equipment fault diagnosis. Lin and Chen [22] found the interesting crossover properties in vibration signals captured from gearboxes and rolling bearings. The scale exponents corresponding to different time scales in double logarithm plots were used as the feature parameters to describe the defective conditions of gears and rolling bearing. Liu [2] claimed that the DFA curves of bearing's vibration signals can be quantified by two scale exponents and the exponents in a small time scale can be utilized to distinguish the faulty bearing conditions. The author's previous research also showed that the gear vibration signals had crossover phenomenon. The scale exponents and intercepts of DFA curves were used for gear fault classification [23]. Jiang et al. [24] evaluated the optimal scaling intervals with Quasi-Monte Carlo algorithm and the least square support vector machine was used for multifault diagnosis of gearbox. Hough [25] combined the least squares method with sliding window to extract the scale exponent and the neural network algorithm was used for classification of gear fault.

However, the detail reasons for multiscales of fault vibration signals were not discussed in abovementioned description. Furthermore, as far as the authors know, the influence of detrend order of DFA on fault recognition was not discussed in previous literatures, and only limited methods for evaluating the crossover points of DFA were developed. In addition, the scale exponents of different time scale intervals were used as the characteristic parameter in previous researches. However, the intercept of double logarithmic plot of the DFA was not utilized. Acutally, the intercept that is used in our research involves a lot of information of vibration signal.

In this paper, the detrended fluctuation analysis (DFA) is employed to analyze the gear vibration signals. According to the double logarithmic plot of the DFA, it is verified that the gear vibration signals exhibit self-similarity in two ranges of time scales. The reason for the double-scale characteristic is discussed through the simulation analysis. Furthermore, the scale exponents and intercepts corresponding to different scale intervals are extracted as the feature vectors to describe the fault condition of gears. It is found that more pieces of information about the gear faults are involved in the small time scale interval. In order to detect the crossover point of two time scales and extract the parameters (scale exponents and intercepts) automatically, a new approach based on the Hough transform is proposed. The experiments were performed with the proposed parameters to classify the gear faults. Combining the Gaussian mixture model (GMM) and Bayesian maximum likelihood classifiers, the classification of gear vibration signals achieved successfully.

The remainder of the paper is organized as follows. Sections 2 and 3 overview the detrended fluctuation analysis (DFA) and the Hough transform (HT). By applying several gear fault simulated signals, the analysis and discussion about the DFA are presented in Section 4. In Section 5, a selfadaptive feature extraction and classification method for the vibration signals based on DFA and HT is introduced and verified by the experiments. Finally, Section 6 contains the conclusions.

\section{Detrended Fluctuation Analysis}

Considering $x(t) t=1,2,3, \ldots, N$ is a time series of length N.

Step 1. Map $x(t)$ to time series $y(m)$ by integration:

$$
y(m)=\sum_{t=1}^{m}[x(t)-\bar{x}] \quad m=1,2,3, \ldots, N,
$$

where $\bar{x}$ is the mean of the time series $x(t)$ :

$$
\bar{x}=\frac{1}{N} \sum_{t=1}^{N} x(t) .
$$

Step 2. Divide $y(m)$ into $N_{s}=\operatorname{int}[N / s]$ which are sub-time series with equal length $s$. The length $N$ of a time series is usually not a multiple of the length $s$, and redundant data of the time series $y(m)$ may be left. Although the redundant data can be deleted in the following analysis, we suggest repeating the same process from the opposite end of the same time series. For each sub-time series, compute the corresponding least squares $p$ order fits:

$$
y_{k}(m)=\sum_{j=0}^{p} \beta_{j} t^{j} \quad\left(k=1,2,3, \ldots, 2 N_{s}\right),
$$

where $y_{k}(m)$ is the trend of the $k$ th sub-time series. It is the fitting polynomial in this sub-time series. Linear, quadratic, cubic, or higher-order polynomials can be used in the fitting procedure (usually called DFA1, DFA2, DFA3, etc.). $\beta_{j}$ is the coefficient of $j$ th order.

Step 3. For each sub-time series, compute the fluctuation function:

$$
F(s)=\left(\frac{1}{2 N_{s}} \sum_{k=1}^{2 N_{s}}\left[F^{2}(s, k)\right]\right)^{1 / 2},
$$


where

$$
\begin{gathered}
F^{2}(s, k)=\frac{1}{s} \sum_{i=1}^{s}\left\{y[(k-1) s+1]-y_{k}(m)\right\}^{2} \\
\text { if } k=1,2, \ldots, N_{s} \\
F^{2}(s, k)=\frac{1}{s} \sum_{i=1}^{s}\left\{y\left[N-\left(k-N_{s}\right) s+i\right]-y_{k}(m)\right\}^{2} \\
\text { if } k=N_{s}+1, \ldots, 2 N_{s} .
\end{gathered}
$$

Step 4. Repeat Steps 1 through 3 for a broad range of sub-time series (i.e., box) with length $s$. If the time series are long-range power-law correlated, the relationship between $F(s)$ and $s$ can be described as follows:

$$
\begin{aligned}
& F(s) \sim s^{\alpha} \Longrightarrow \\
& F(s)=A s^{\alpha},
\end{aligned}
$$

where $\alpha$ is the scale exponent. It can be calculated by taking the logarithm of both sides of (6),

$$
\log (F(s))=\log A+\alpha \log s,
$$

and subsequently plotting $\log (F(s))$ versus $\log s$ to obtain scale exponent $\alpha$ and intercept $\log A$ by linear regression.

The scale exponent $\alpha$ characterizes the long-range powerlaw correlation properties of the time series. It has a close relationship with the self-correlation function. If $\alpha=0.5,1$, and 1.5 , the characteristics of the time series correspond to the independent random process (white noise), $1 / f$ process, and Brownian motion, respectively. If $0<\alpha<0.5$, the correlations in the signal are antipersistent (negative correlations). If $0.5<\alpha \leq 1$, correlations in the signal are persistent (positive correlations).

\section{Hough Transform}

The Hough transform [25] is an automatic image analysis technique which can be used to detect regular curves such as straight lines, circles, and ellipses within an image. The plotting of $\log (F(s))$ versus $\log s$ of DFA can be seen as an image. The linear relationship between $\log (F(s))$ and $\log s$ means a series of straight lines in the plotting. The Hough transform for detecting straight lines is introduced as follows.

Generally, in a Cartesian coordinate plane $(X, Y)$, a straight line can be described as $y=a x+b$, where parameters $a$ and $b$ are the slope and intercept, respectively. Only when the values of $a$ and $b$ are known, can we describe this line accurately. The point $\left(x_{0}, y_{0}\right)$ on this line can be written as $y_{0}=a x_{0}+b$ and it can be changed as $b=-x_{0} a+y_{0}$, which indicate a straight line in the coordinate plane $(A, B)$. That means a point in plane $(X, Y)$ corresponds to a line in plane $(A, B)$ and vice versa. If every point on a line $y=$ $a x+b$ in plane $(X, Y)$ is mapped to plane $(A, B)$, the lines will cross at one point $(a, b)$ and the line in plane $(X, Y)$ will be identified. If there are several crossed points in plane

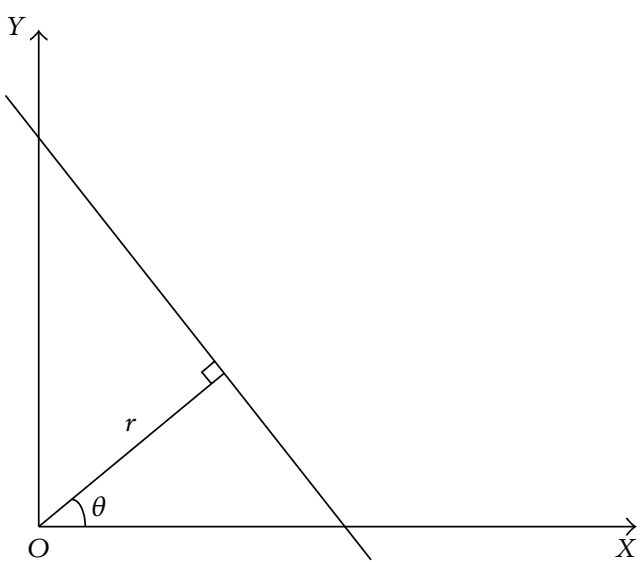

FIGURE 1: The pair of parameters $(r, \theta)$ used in polar coordinates was used to replace the pair of parameters $(a, b)$ used in Cartesian coordinates as the Hough parameter space.

$$
\begin{aligned}
& \text { Initialize accumulator } H(r, \theta) \text { to all zeros; } \\
& \text { For each point }\left(x_{i}, y_{i}\right) \text { in the }(X, Y) \text { plane, } \\
& \text { For } \theta=0 \text { to } 180 \\
& r=x_{i} \cos \theta+y_{i} \sin \theta \\
& \quad H(r, \theta)=H(r, \theta)+1 \\
& \text { End } \\
& \text { End }
\end{aligned}
$$

Algorithm 1

$(A, B)$, several straight lines will be identified in plane $(X, Y)$. However, vertical lines in the $(X, Y)$ plane described as $x=a$ will give rise to unbounded values of the slope parameter $a$. Thus, Duda and Hart proposed the use of a different pair of parameters $(r, \theta)$ used in polar coordinates, which are referred to as the Hough parameter space, to replace the pair of parameters $(a, b)$ used in Cartesian coordinates (Figure 1). Consider

$$
r=x \cos \theta+y \sin \theta .
$$

The outline of the Hough transform consists of the steps shown in Algorithm 1.

Find the values of $\left(r_{i}, \theta_{i}\right)$, where $H(r, \theta)$ is a local maximum.

The detected lines in the $(x, y)$ plane will be $r_{i}=x \cos \theta_{i}+$ $y \sin \theta_{i}$.

\section{Detrended Fluctuation Analysis of Simulated Signals and Discussion}

Different vibration condition signals of gears contain different frequency components and amplitudes. The main frequencies that should be paid more attention include rotation frequency, meshing frequencies, their harmonic frequencies, and sidebands. Usually, the gear's vibration signal can be regarded as a combination of a series of sinusoid signals with different frequencies and random noise. 


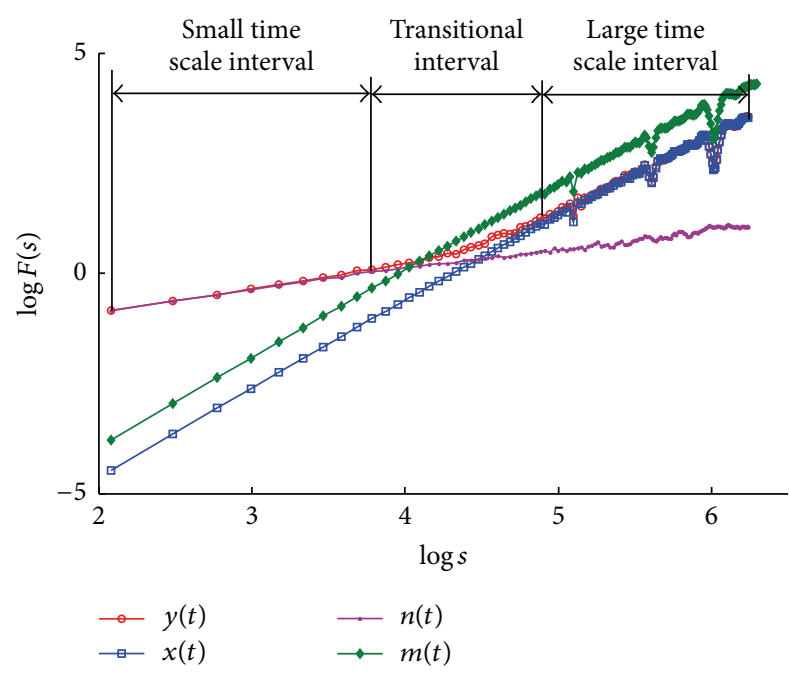

FIGURE 2: $\log F(s)$ versus $\log s$ of $y(t), x(t), n(t)$, and $m(t)$.

Consider the composite signal $y(t)=x(t)+n(t)$ and $m(t)=2 x(t)$, where $n(t)$ is a Gaussian distributed random signal and $x(t)=0.8 \sin (10 \pi t)+0.1 \sin (20 \pi t)+0.1 \sin (40 \pi t)$.

The signal-noise-ratio (SNR) of $y(t)$ is equal to 2.84 . The definition of SNR is as follows:

$$
\mathrm{SNR}=10 \lg \frac{\|x\|}{\|e\|},
$$

where $\|x\|$ and $\|e\|$ are the root-mean-squares of $x(t)$ and $n(t)$, respectively.

The logarithm scale fluctuation function maps of $y(t)$, $x(t), m(t)$, and $n(t)$ are shown in Figure 2 .

Figure 2 shows that the correlations of simulated data $y(t)$ do not follow the same scaling law in all time scales. Obviously, there are two linear intervals and a transitional interval in a double logarithmic plot of the DFA for mixtured signal $y(t)$. Comparing $y(t)$ with $n(t)$ and $x(t)$, in a small time scale interval, $y(t)$ shows similar linear features with random signal $n(t)$, which corresponds to local fluctuation and high frequency components. In a large time scale interval, it shows similar features with periodic signal $x(t)$ which corresponds to the large fluctuation and low frequency periodic components. Since the gear's vibration signal can be regarded as a combination of a series of periodic signals with different frequencies and random noise, the gear's vibration will present double-scale characteristic too.

Moreover, comparing the double logarithmic plot of $x(t)$ and $m(t)$, the scale exponents are the same. However, the intercept of $m(t)$ is larger than the intercept of $x(t)$ because the amplitude of $m(t)$ is larger than that of $x(t)$. Therefore, the intercept is a useful parameter which characterizes the signal intensity.

For the gear vibration signal, its characteristics analyzed by DFA are correlated with the fault conditions. Different fault patterns will cause different scale exponents. Moreover, a more severe defect will cause a larger vibration intensity, which will cause a larger intercept of double logarithmic plot.

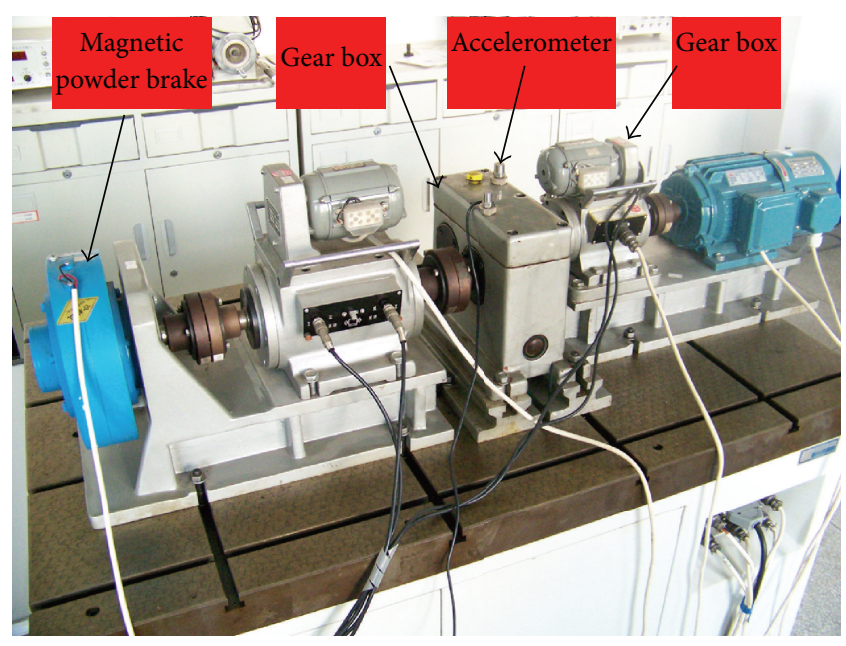

FIGURE 3: The experimental setup of the gearbox fault detection.

In our research, the scale exponent $\alpha$ and intercept $b$ of different time scale are utilized as the characteristic parameters to describe the gear vibration conditions. The aforementioned Hough transform is used to locate the position of crossover point and distinguish linear relation of small and large time scales and to get correct scale exponent and intercept.

\section{Application of DFA to Gearbox Fault Diagnostics}

In this section, the signals corresponding to four gear fault conditions obtained from gearbox experimental facility are analyzed by the DFA. The scale exponent and intercept are extracted as characteristic parameters to describe the gear conditions. Combining Gaussian mixture model (GMM) with Bayesian maximum likelihood classifier, these signals are classified.

5.1. Experimental Setup. The experiment setup is shown in Figure 3 and its schematic diagram is shown in Figure 4 . The experimental facility consists of an electric machine, a single stage gearbox with a pair of spur gears, a magnetic powder brake with necessary load, and an I/O Tech Wave Book/516E 16-bit $1 \mathrm{MHz}$ data acquisition system with Ethernet interface. A $0.55 \mathrm{~kW}$ DC motor rotates the pinion which has 20 teeth and the mating gear which has 37 teeth is loaded by a magnetic powder brake. The vibration generated by the gearbox was picked up by a PCB piezoelectric vibration accelerometer. The accelerometer is mounted on vertical direction of the bearing block at input end. For each kind of gear failures, including "Normal," "Toothless," "Scratched," and "Circular pitch error", 150 groups of vibration signals are acquired. The motor's rotational speed and transferred load were random values which lie in the ranges of $300 \mathrm{r} / \mathrm{min}-$ $1217 \mathrm{r} / \mathrm{min}$ and $0 \mathrm{~N} \cdot \mathrm{m}-20 \mathrm{~N} \cdot \mathrm{m}$, respectively. The sampling frequency is $10 \mathrm{kHz}$. 


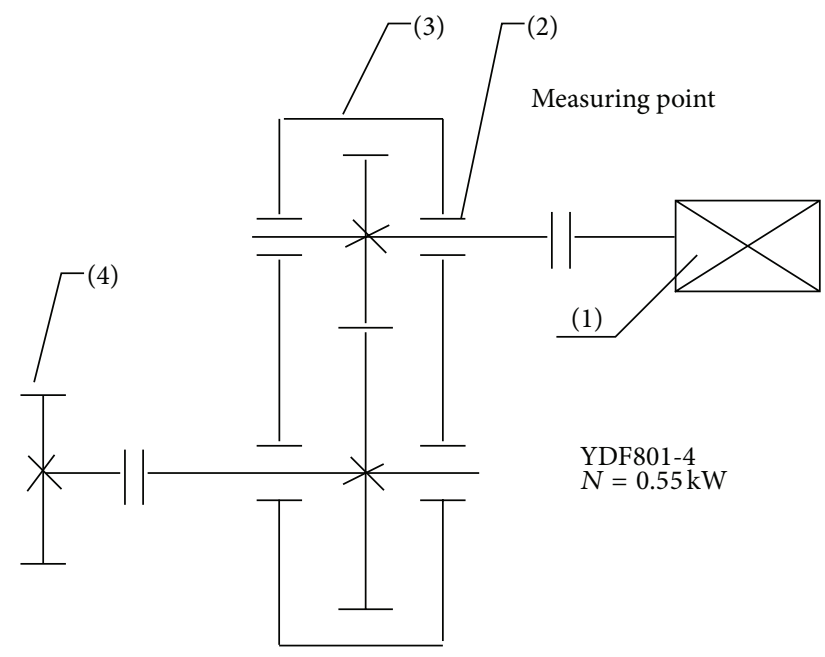

(1) Motor

(2) Acceleration transducer

(3) Single geared drive box

(4) Magnetic powder brake

FIGURE 4: The schematic diagram of the experimental setup.

5.2. Signals and Discussion. The representative vibration signal and $\log (F(s))$ versus $\log s$ map of "Normal" and "Toothless" gear conditions are shown in Figure 5. The detrend order is one and the minimum and maximum window sizes are 8 and 512 sample points, respectively. For captured vibration signals, Figures 5(c) and 5(d) show that there are two different scaling intervals in the double logarithmic plot of DFA fluctuation function. As we discussed in Section 4, the gear vibration signals can be seen as the combination of random noise and a series of period signals which have different amplitudes and frequencies. The interval of small time scales corresponds to a random signal which includes local fluctuation and high frequency components, and the large one corresponds to the periodic components in the signal which are well correlated.

Consider the double logarithmic plot as a binary image on which the values of pixels at a given coordinate, $(\log (s), \log [F(s)])$, are one and the values of the rest of the pixels are equal to zero. With Hough transform, the strange line corresponding to two ranges will be detected. Accordingly, the corner point, the scale exponent $\alpha$, and the intercept $b$ in different time scale will be extracted automatically. A proposed feature vector mapping of vibration signals is shown in Figure 6.

As Figure 6(a) shows, the characteristic parameter maps of four kinds of gear faults overlap weakly in the small time scale.

Except for the "Circular pitch error", there is a small overlap between the "Normal," "Scratched," and "Toothless" gears. In the large time scale, the maps of "Scratched" and "Toothless" gears are overlapped completely, which indicates that the clustered results are better in the small time scale than those in large time scale. In theory, even if the gear has a tiny defect or the gear fault condition changes slightly, the vibration condition caused by the reduction of meshing stiffness will change. However, these changes may be so subtle that only local fluctuation in signals is affected. The local fluctuation of signals corresponds to signals' morphological characters in small time scales interval or high frequency components rather than the large time scales interval or low frequency components. Only when the severity of defects reaches a lever or the fault conditions change a lot will the variation of large fluctuation in vibration signals and the difference in large time scale intervals be observed. Therefore, comparing to large time scales, there are more useful pieces of diagnostic information in small scales. As a contrast, the characteristic parameter maps of four kinds of gear faults by traditional DFA without considering linear relationship in different time range are shown in Figure 6(c). Obviously, the maps of Scratched and Toothless gears are overlapped mostly. That means that the difference between "Scratched" and "Toothless" conditions signals that cannot be distinguished in traditional DFA can be identified by small time scale parameters in double-scale logarithmic plot. There is more useful diagnostic information in small scales. In the following section, the feature vector consisting of scale exponent $\alpha$ and intercept $b$ in small time scale is used to characterize the gear fault vibration signal. The Gaussian mixture model and maximum Bayes classification will be employed to identify the gear faults.

\subsection{Fault Recognition Algorithms Combined Gaussian Mixture} Model with DFA. Considering we have a training dataset and a testing dataset which consist of a series of gearbox vibration signals, the main steps of the proposed algorithm for gear fault classification are described as follows.

Step 1. For a kind of fault condition, employ DFA to plot the double logarithm graphs of all training signals and extract the feature vector $\left(\alpha_{1}, b_{1}\right)$ of small time scale as training space by Hough transform, where $\alpha_{1}$ is scale exponents and $b_{1}$ is intercept of double logarithm graphs.

Step 2. Build the Gaussian mixture model (GMM) of training space corresponding to this kind of fault by expectation maximum algorithm. The GMM is defined by

$$
p(x)=\sum_{k=1}^{M} w_{k} p_{k}(x)=\sum_{k=1}^{M} w_{k} N\left(x ; \mu_{k}, \Sigma_{k}\right),
$$

where $M$ is the number of mixtures, $w_{k}$ is the mixture weight with the constraint that $\sum w_{k}=1$, and $x=\left(\alpha_{1}, b_{1}\right)$. $N\left(x ; \mu_{k}, \Sigma_{k}\right)=\left(1 /(2 \pi)^{1 / 2}\left|\Sigma_{k}\right|^{1 / 2}\right) e^{-(1 / 2)\left(x-\mu_{k}\right)^{T} \Sigma_{k}^{-1}\left(x-\mu_{k}\right)}$ is Gaussian probability function which describes the $k$ th normal distribution, such as center, width, and direction [26], with mean $\mu_{k}$ and covariance matrix $\Sigma_{k}$.

Step 3. Repeat Steps 1 and 2 to build GMMs for all kinds of gear fault condition signals.

Step 4. Before a testing signal is classified, the feature vector $\left(\alpha_{2}, b_{2}\right)$ should be extracted as mentioned in Step 1. Then, the signal is classified using a Bayesian maximum likelihood classifier. This is accomplished by computing the conditional 


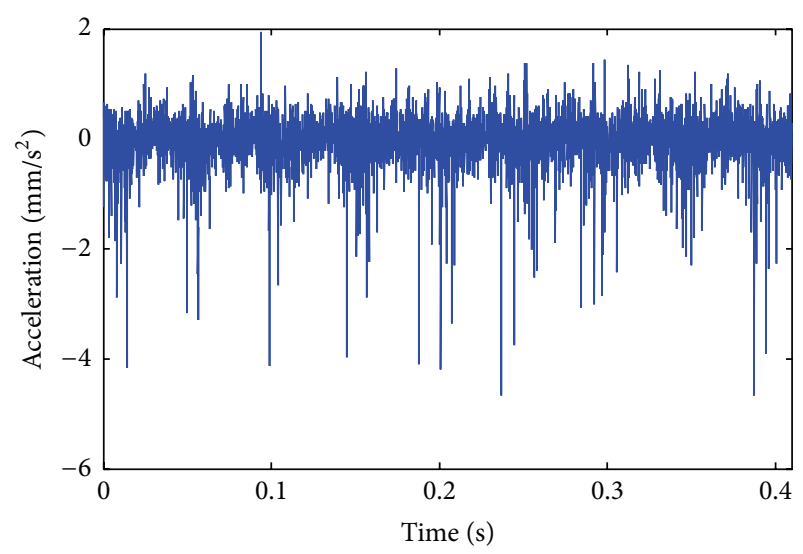

(a)

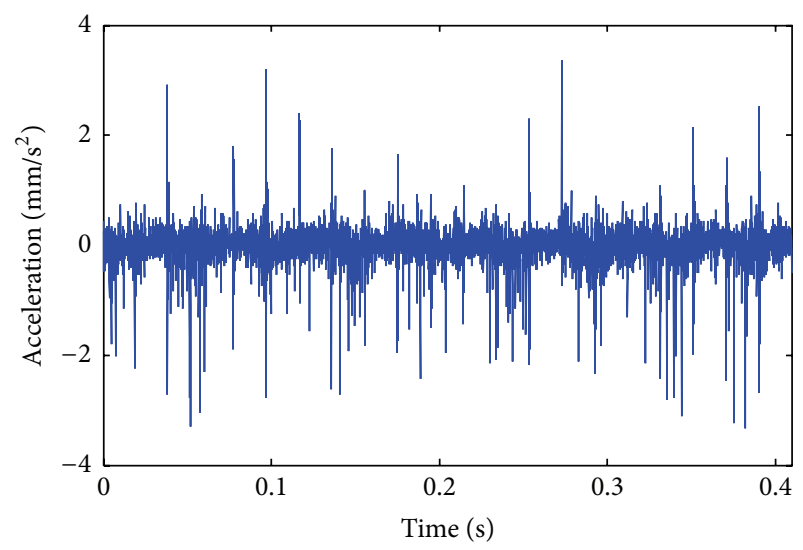

(c)

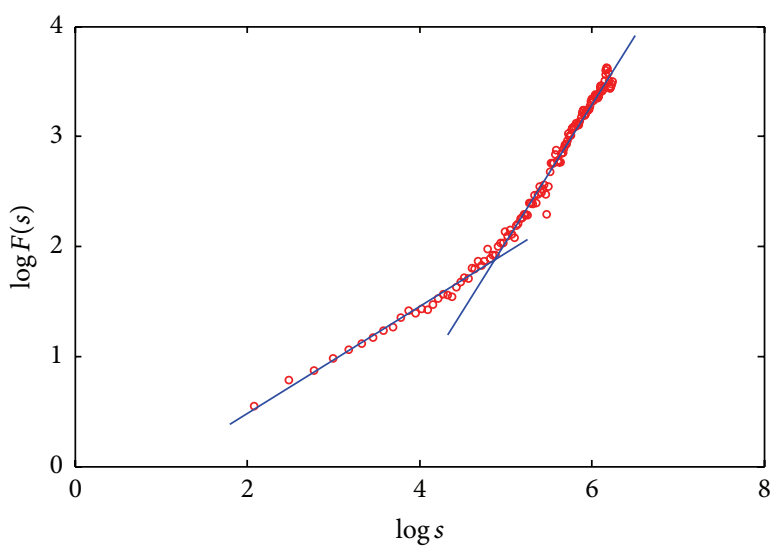

(b)

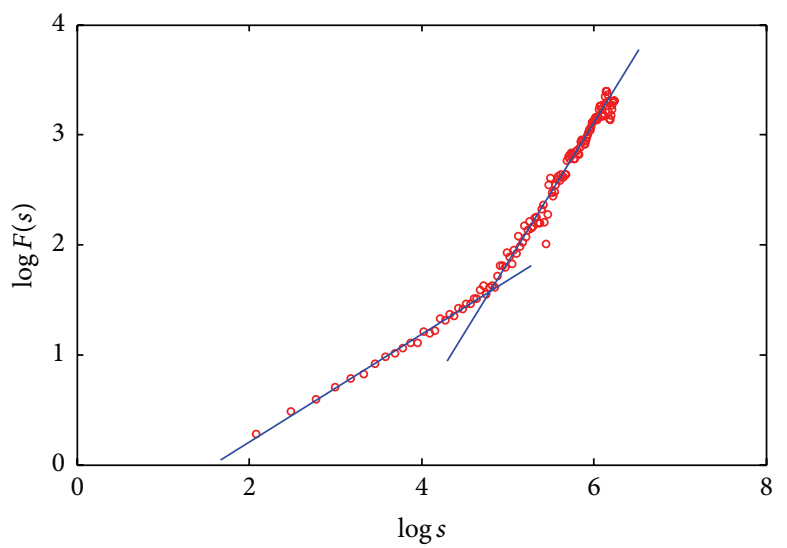

(d)

FIGURE 5: The representative vibration signal, power spectrum, and the DFA curves obtained from the two types of gears with a rotation speed of 985 rpm: (a) Signal from a "Normal" gear. (b) DFA from a "Normal" gear. (c) Signal from a "Toothless" gear. (d) DFA from a "Toothless" gear.

likelihoods of the signal under each learned GMM and by selecting the model with the highest likelihood:

$$
\widehat{c}=\arg \max p\left(Y \mid c_{i}\right)
$$

where $Y$ is the feature vector $(\alpha, b)$ of the testing signal and $p\left(Y \mid c_{i}\right)$ is the probability of $Y$ with known $i$ th gear fault condition described by $i$ th GMM.

In our classification experiment, for each gear condition, 100 signals were selected to constitute training dataset and 50 signals were selected to constitute testing dataset to verify the proposed approach. The minimum and maximum window sizes are 8 and 512 sampling points, respectively, and the mixture number of GMM is four. In order to evaluate the influence of the trend order of DFA which may change the position of crossover point, the classification experiments, when detrend order of DFA ranges from one to six, are conducted and the classification results are listed in Tables 1-6. The plotting of recognition rate versus trend order of DFA is shown in Figure 7.

Tables 1-6 show that, for the "Normal," "Scratched," and "Circular pitch error" signals, the recognition rates are
TABLE 1: Classification results with proposed method (DFA1).

\begin{tabular}{lccccc}
\hline \multirow{2}{*}{ Gear fault } & \multicolumn{5}{c}{ Diagnosis results } \\
& NOR & SCR & TL & CPE & Recognition rate \\
\hline NOR & 49 & 0 & 1 & 0 & $98 \%$ \\
SCR & 0 & 46 & 4 & 0 & $92 \%$ \\
TL & 5 & 3 & 42 & 0 & $84 \%$ \\
CPE & 0 & 0 & 0 & 50 & $100 \%$ \\
\hline
\end{tabular}

NOR: "Normal"; SCR: "Scratched"; TL: "Toothless"; CPE: "Circular pitch error."

TABLE 2: Classification results with proposed method (DFA2).

\begin{tabular}{lccccc}
\hline \multirow{2}{*}{ Gear fault } & \multicolumn{5}{c}{ Diagnosis results } \\
& NOR & SCR & TL & CPE & Recognition rate \\
\hline NOR & 50 & 0 & 0 & 0 & $100 \%$ \\
SCR & 0 & 45 & 5 & 0 & $90 \%$ \\
TL & 6 & 2 & 42 & 0 & $84 \%$ \\
CPE & 0 & 0 & 0 & 50 & $100 \%$ \\
\hline
\end{tabular}

over 90 percent; even the detrend order of DFA is changed. The recognition rate of "Toothless" signal is less than 90 


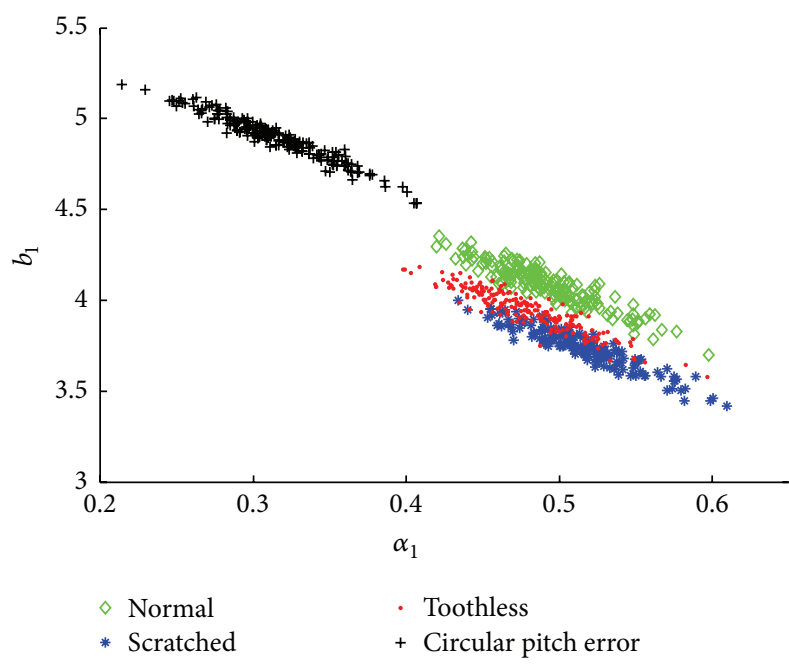

(a)

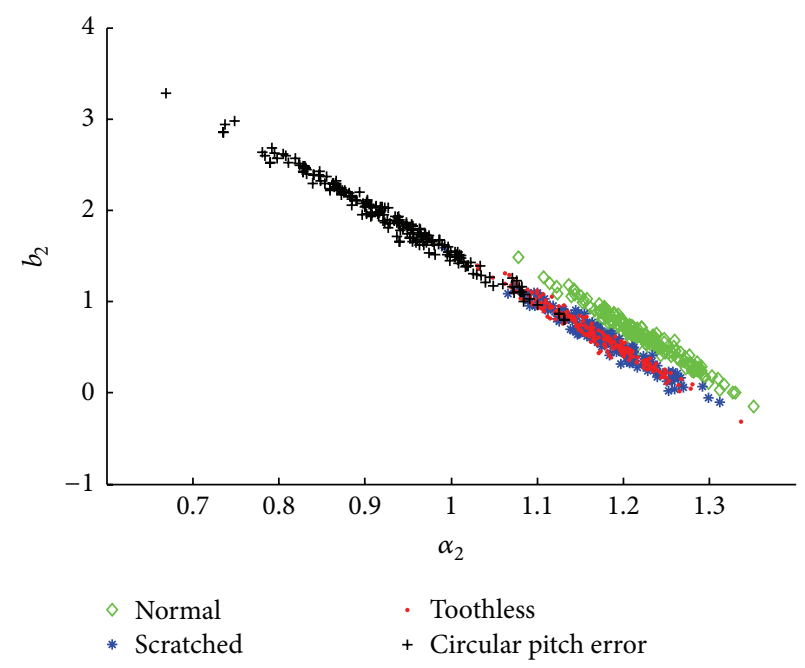

(b)

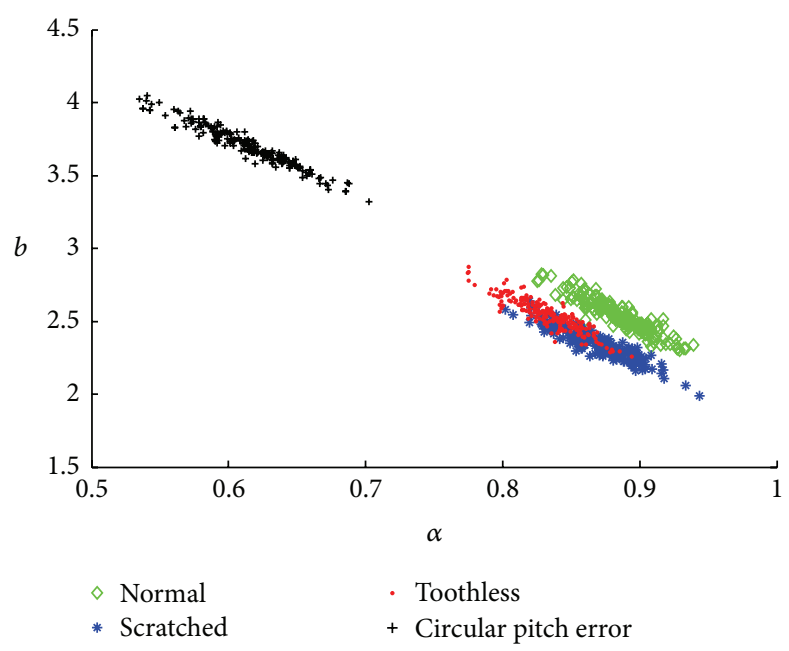

(c)

FIGURE 6: $(\alpha, b)$ map of different ranges of scales: (a) $\alpha_{1}$ and $b_{1}$ in the first range of scales; (b) $\alpha_{2}$ and $b_{2}$ in the second range of scales; (c) $\alpha$ and $b$ extracted by traditional DFA.

TABLE 3: Classification results with proposed method (DFA3).

\begin{tabular}{lccccc}
\hline \multirow{2}{*}{ Gear fault } & \multicolumn{5}{c}{ Diagnosis results } \\
& NOR & SCR & TL & CPE & Recognition rate \\
\hline NOR & 49 & 0 & 0 & 1 & $98 \%$ \\
SCR & 0 & 48 & 2 & 0 & $96 \%$ \\
TL & 7 & 1 & 42 & 0 & $84 \%$ \\
CPE & 0 & 0 & 0 & 50 & $100 \%$ \\
\hline
\end{tabular}

percent in all the classification experiments. Few of samples of "Scratched" and "Toothless" signals are misclassified as the "Normal." Theoretically, if we study the signals from the frequency domain, the differences between "Normal" gears, "Scratched" gears, and "Toothless" gears are the vibration amplitudes on some special frequencies, such as rotation frequency, meshing frequency, and their frequency multiplication. With the severity of gear fault increasing, the vibration
TABLE 4: Classification results with proposed method (DFA4).

\begin{tabular}{lccccc}
\hline \multirow{2}{*}{ Gear fault } & \multicolumn{5}{c}{ Diagnosis results } \\
& NOR & SCR & TL & CPE & Recognition rate \\
\hline NOR & 46 & 0 & 3 & 0 & $92 \%$ \\
SCR & 0 & 49 & 1 & 0 & $98 \%$ \\
TL & 10 & 1 & 39 & 0 & $78 \%$ \\
CPE & 0 & 0 & 0 & 50 & $100 \%$ \\
\hline
\end{tabular}

amplitude will increase slowly and the noise will eventually cause the fault characteristic overlap of three kinds of gears' vibration signals. That causes the identification of these three kinds of fault conditions difficult in our experiments. Figure 7 shows that when detrend order of DFA changes from one to six, the recognition rate of "Normal," "Scratched," and "Circular pitch error" signals keeps the range from 90 precent to 100 precent; however, the global trend of recognition 
TABLE 5: Classification results with proposed method (DFA5).

\begin{tabular}{lccccc}
\hline \multirow{2}{*}{ Gear fault } & \multicolumn{5}{c}{ Diagnosis results } \\
& NOR & SCR & TL & CPE & Recognition rate \\
\hline NOR & 45 & 1 & 3 & 1 & $90 \%$ \\
SCR & 0 & 47 & 3 & 0 & $94 \%$ \\
TL & 10 & 1 & 39 & 0 & $78 \%$ \\
CPE & 0 & 0 & 0 & 50 & $100 \%$ \\
\hline
\end{tabular}

TABLE 6: Classification results with proposed method (DFA6).

\begin{tabular}{lccccc}
\hline \multirow{2}{*}{ Gear fault } & \multicolumn{5}{c}{ Diagnosis results } \\
& NOR & SCR & TL & CPE & Recognition rate \\
\hline NOR & 46 & 0 & 4 & 0 & $92 \%$ \\
SCR & 1 & 46 & 3 & 0 & $92 \%$ \\
TL & 14 & 4 & 32 & 0 & $64 \%$ \\
CPE & 1 & 0 & 0 & 49 & $98 \%$ \\
\hline
\end{tabular}

rate is decreasing with the increasing of detrend order of DFA. The recognition rate of "Toothless" signals will decrease monotonically with the detrend order of DFA increase. That means that the detrend order of DFA influences the classification results. Higher detrend order of DFA may cause less recognition rate. As a suggestion, the detrend order of DFA should be low in order to get better classification result.

\section{Conclusion}

In this study, the detrended fluctuation analysis (DFA) which can deal with nonstationary signals was employed to analyze the gear vibration signals acquired by piezoelectric acceleration transducer and the experimental results showed that the characteristics of all the gear condition signals turned out to be double-scale. To further understand the phenomenon of the double-scale, the experimental signals were analyzed by simulation. The simulation results show that the doublescales correspond to high and low frequency components of signal, respectively, and the intercepts are determined by signal intensity. A feature vector which employed an exponent $\alpha$ and an intercept $b$ in small time scale was proposed to describe slight defect of gear. Since the vibration pattern caused by the reduction of meshing stiffness changes local fluctuation of vibration signal subtly due to a slight defect, it is more suitable to use double-scale parameters for gear conditions classification instead of the traditional DFA. In addition, the Hough transform was used to estimate the position of crossover point and extract the scale exponent and intercept of the small time scale from the double logarithmic plot of DFA automatically. Moreover, the Gaussian mixture model (GMM) and Bayesian maximum likelihood (BML) classifier were employed to describe the distribution of the feature vector and identify the patterns of test data, respectively. The classification experiments with different detrend order of DFA were conducted. The experimental results demonstrate that the proposed approach is the effectiveness and the detrend order of DFA sholud be low for more accurate classification results.

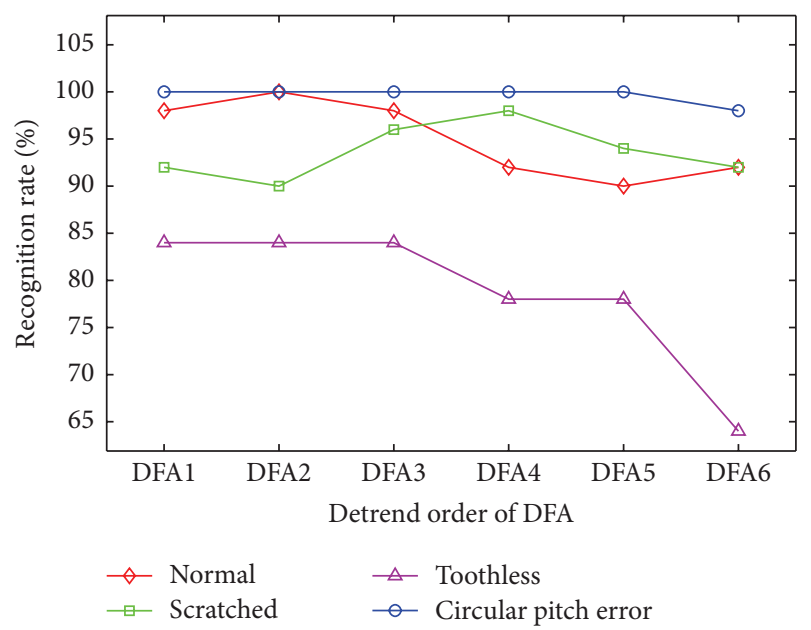

FIGURE 7: The plotting of recognition rate versus trend order of DFA.

\section{Conflict of Interests}

The authors declare that there is no conflict of interests regarding the publication of this paper.

\section{Acknowledgment}

The reported research was partially based upon work supported by National Natural Science Foundation of China (NSFC) awards (Grant nos. 51105284, 51475339, 51375154, and 51405353).

\section{References}

[1] S. J. Loutridis, "Self-similarity in vibration time series: application to gear fault diagnostics," Journal of Vibration and Acoustics, vol. 130, no. 3, Article ID 031004, 2008.

[2] J. Liu, "Detrended fluctuation analysis of vibration signals for bearing fault detection," in Proceedings of the IEEE International Conference on Prognostics and Health Management (PHM '11), pp. 1-5, IEEE, Montreal, Canada, June 2011.

[3] J. Wang, R. Li, and X. Peng, "Survey of nonlinear vibration of gear transmission systems," Applied Mechanics Reviews, vol. 56, no. 3, pp. 309-329, 2003.

[4] R. Bajrić, D. Sprečić, and N. Zuber, "Review of vibration signal processing techniques towards gear pairs damage identification," International Journal of Engineering \& Technology IJETIJENS, vol. 11, no. 4, pp. 124-128, 2011.

[5] X. Wang, C. Liu, F. Bi, X. Bi, and K. Shao, "Fault diagnosis of diesel engine based on adaptive wavelet packets and EEMDfractal dimension," Mechanical Systems and Signal Processing, vol. 41, no. 1-2, pp. 581-597, 2013.

[6] L. Zhang, G. Xiong, H. Liu, H. Zou, and W. Guo, "Bearing fault diagnosis using multi-scale entropy and adaptive neuro-fuzzy inference," Expert Systems with Applications, vol. 37, no. 8, pp. 6077-6085, 2010.

[7] L.-Y. Wang, W.-G. Zhao, and D.-H. Wei, "Application of Lyapunov exponent spectrum in pressure fluctuation of draft tube," Journal of Hydrodynamics. Series B, vol. 21, no. 6, pp. 856-860, 2009. 
[8] J. W. Kantelhardt, S. A. Zschiegner, E. Koscielny-Bunde, S. Havlin, A. Bunde, and H. E. Stanley, "Multifractal detrended fluctuation analysis of nonstationary time series," Physica A: Statistical Mechanics and Its Applications, vol. 316, no. 1-4, pp. 87-114, 2002.

[9] J. B. Bassingthwaighte and G. M. Raymond, "Evaluating rescaled range analysis for time series," Annals of Biomedical Engineering, vol. 22, no. 4, pp. 432-444, 1994.

[10] C.-K. Peng, S. V. Buldyrev, A. L. Goldberger et al., "Long-range correlations in nucleotide sequences," Nature, vol. 356, no. 6365, pp. 168-170, 1992.

[11] K. Koçak, "Examination of persistence properties of wind speed records using detrended fluctuation analysis," Energy, vol. 34, no. 11, pp. 1980-1985, 2009.

[12] J. M. O. Matos, E. P. de Moura, S. E. Krüger, and J. M. A. Rebello, "Rescaled range analysis and detrended fluctuation analysis study of cast irons ultrasonic backscattered signals," Chaos, Solitons \& Fractals, vol. 19, no. 1, pp. 55-60, 2004.

[13] J. C. Reboredo, M. A. Rivera-Castro, J. G. V. Miranda, and R. García-Rubio, "How fast do stock prices adjust to market efficiency? Evidence from a detrended fluctuation analysis," Physica A: Statistical Mechanics and Its Applications, vol. 392, no. 7, pp. 1631-1637, 2013.

[14] J. C. Echeverría, J. Álvarez-Ramírez, M. A. Peña, E. Rodríguez, M. J. Gaitán, and R. González-Camarena, "Fractal and nonlinear changes in the long-term baseline fluctuations of fetal heart rate," Medical Engineering \& Physics, vol. 34, no. 4, pp. 466-471, 2012.

[15] M. A. Little and J. P. Bloomfield, "Robust evidence for random fractal scaling of groundwater levels in unconfined aquifers," Journal of Hydrology, vol. 393, no. 3-4, pp. 362-369, 2010.

[16] E. P. de Moura, A. P. Vieira, M. A. S. Irmão, and A. A. Silva, "Applications of detrended-fluctuation analysis to gearbox fault diagnosis," Mechanical Systems and Signal Processing, vol. 23, no. 3, pp. 682-689, 2009.

[17] E. P. De Moura, C. R. Souto, A. A. Silva, and M. A. S. Irmão, "Evaluation of principal component analysis and neural network performance for bearing fault diagnosis from vibration signal processed by RS and DF analyses," Mechanical Systems and Signal Processing, vol. 25, no. 5, pp. 1765-1772, 2011.

[18] M. Sridhar, D. Venkata Ratnam, B. Sirisha et al., "Characterization of low latitude ionospheric scintillations using EEMDDFA method," in Proceedings of the IEEE International Conference on Innovations in Information, Embedded and Communication Systems (ICIIECS '15), pp. 1-6, Coimbatore, India, March 2015.

[19] M. A. Shi-Hao, "Crossover phenomena in detrended fluctuation analysis used in financial markets," Communications in Theoretical Physics, vol. 51, no. 2, pp. 358-362, 2009.

[20] R. G. Kavasseri and R. Nagarajan, "Evidence of crossover phenomena in wind-speed data," IEEE Transactions on Circuits and Systems I: Regular Papers, vol. 51, no. 11, pp. 2255-2262, 2004.

[21] C.-K. Peng, S. Havlin, H. E. Stanley, and A. L. Goldberger, "Quantification of scaling exponents and crossover phenomena in nonstationary heartbeat time series," Chaos, vol. 5, no. 1, pp. 82-87, 1995.

[22] J. Lin and Q. Chen, "A novel method for feature extraction using crossover characteristics of nonlinear data and its application to fault diagnosis of rotary machinery," Mechanical Systems and Signal Processing, vol. 48, no. 1-2, pp. 174-187, 2014.
[23] H. Xiao, Y. Lv, and T. Wang, "Detrended fluctuation analysis to gear's vibration signals and its application in fault classification," Journal of Vibration of Engineering, vol. 28, pp. 331-336, 2015.

[24] X. Jiang, S. Li, and Y. Wang, "1641. A novel method for selfadaptive feature extraction using scaling crossover characteristics of signals and combining with LS-SVM for multi-fault diagnosis of gearbox," Journal of Vibroengineering, vol. 17, no. 4, pp. 1861-1878, 2015.

[25] P. V. C. Hough, "Method and means for recognizing complex patterns," U.S. Patent no. 3,069,654, U.S. Patent and Trademark Office, Washington, DC, USA, 1962.

[26] A. C. Lindgren, M. T. Johnson, and R. J. Povinelli, "Speech recognition using reconstructed phase space features," in Proceedings of the IEEE International Conference on Speech, and Signal Processing (ICASSP '03), vol. 1, pp. I-60-I-63, IEEE, April 2003. 


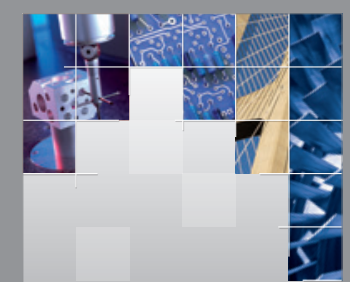

\section{Enfincering}
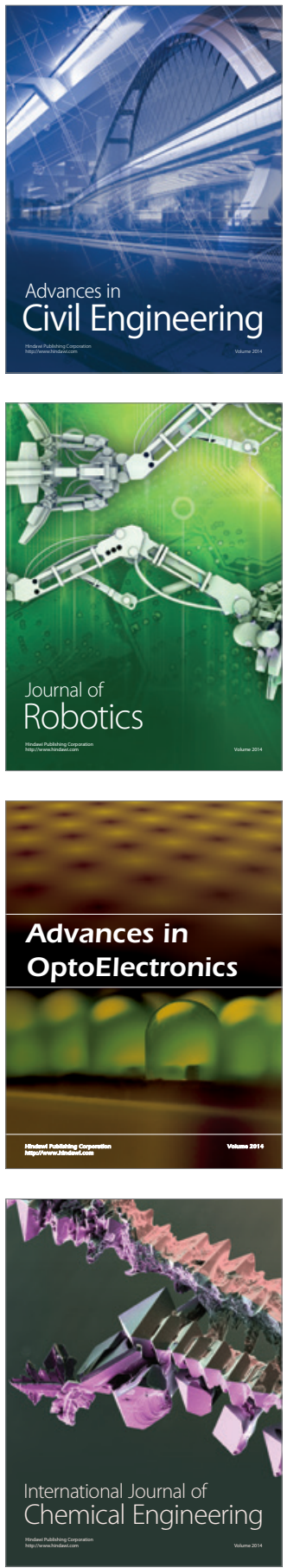

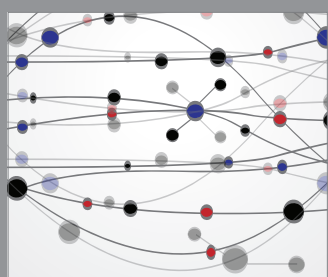

The Scientific World Journal

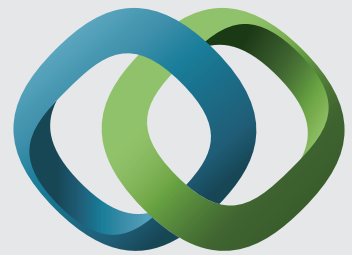

\section{Hindawi}

Submit your manuscripts at

http://www.hindawi.com
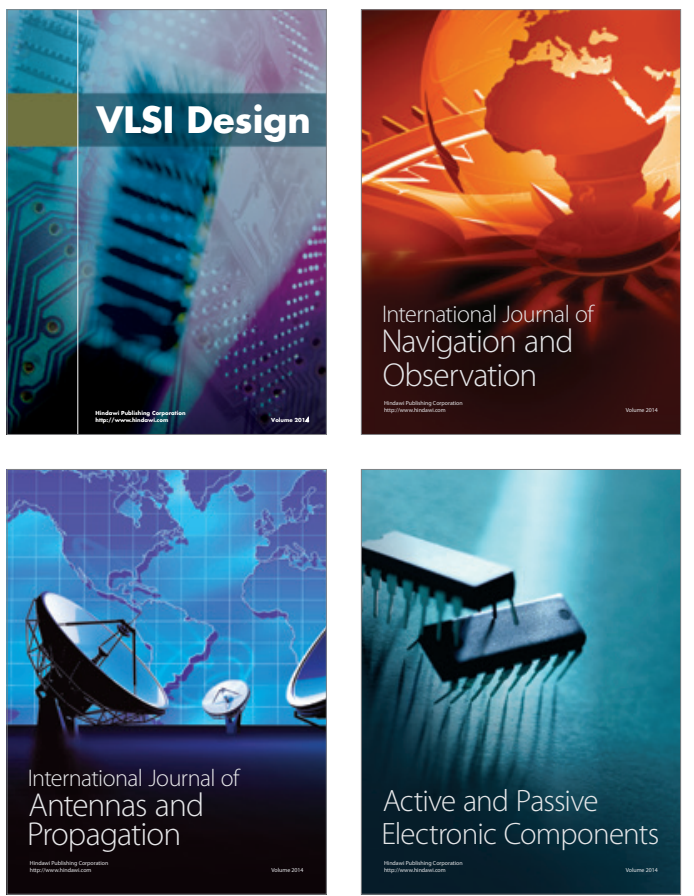
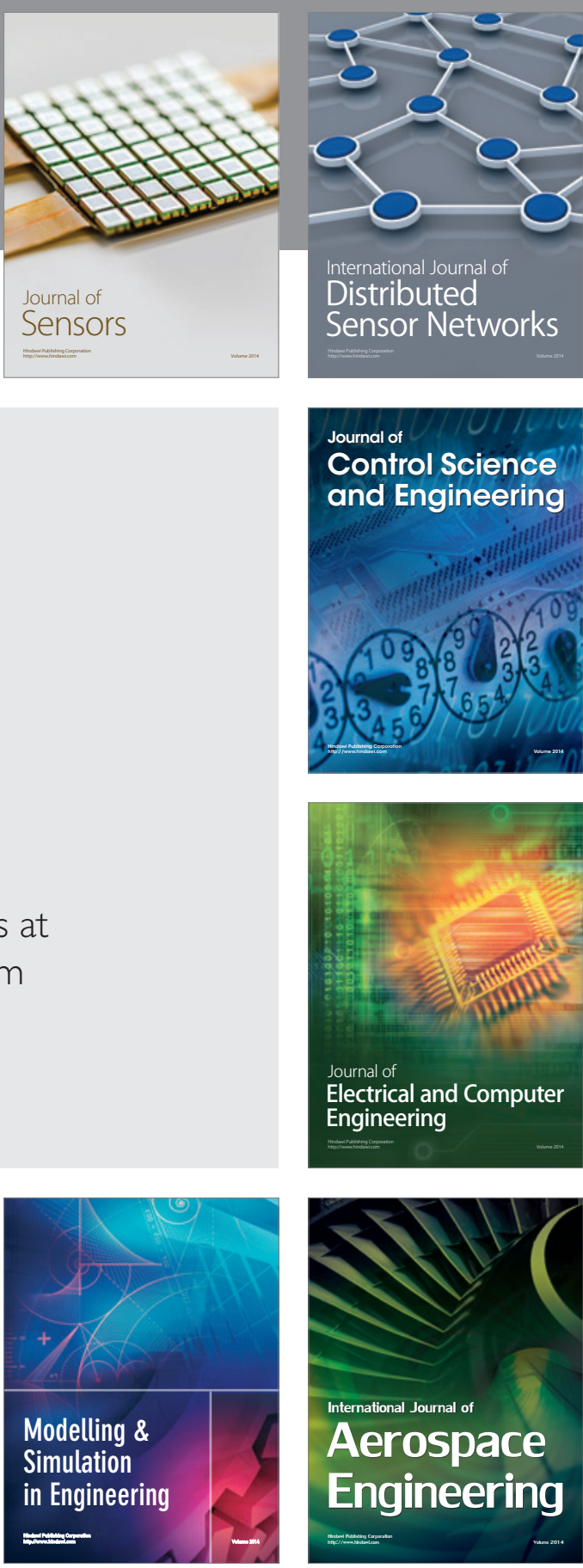

International Journal of

Distributed

Sensor Networks

Journal of

Control Science

and Engineering
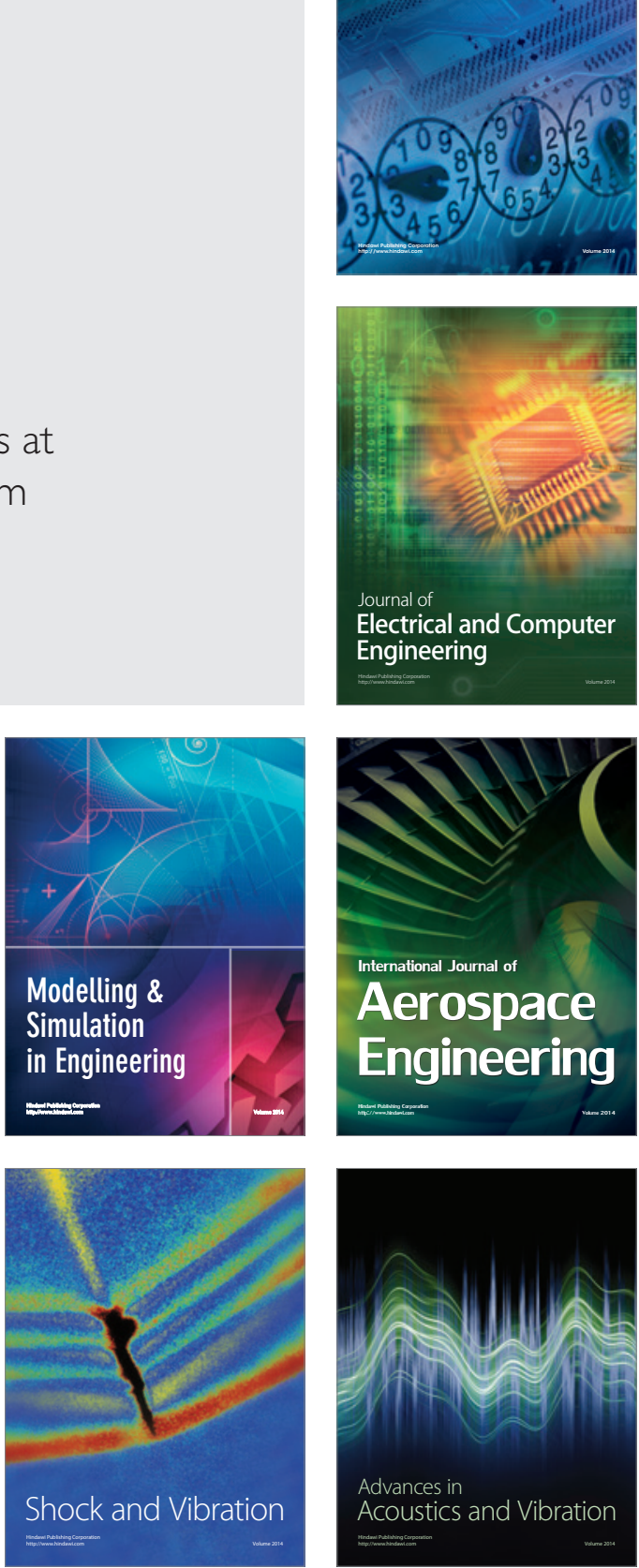